\title{
BMJ Open Physical therapy and orthopaedic equipment-induced reduction in the biomechanical risk factors related to knee osteoarthritis: a systematic review and Bayesian network meta-analysis of randomised controlled trials
}

\author{
Xi-Meng Huang (D) , ${ }^{1}$ Fu-Zhen Yuan, ${ }^{1}$ You-Rong Chen, ${ }^{1}$ Ying Huang, ${ }^{2}$ Ze-Xi Yang, ${ }^{3}$ \\ Lin Lin, ${ }^{1}$ Jia-Kuo $\mathrm{Yu}^{1}$
}

To cite: Huang X-M, Yuan FZ, Chen Y-R, et al. Physical therapy and orthopaedic equipment-induced reduction in the biomechanical risk factors related to knee osteoarthritis: a systematic review and Bayesian network meta-analysis of randomised controlled trials. BMJ Open 2022;12:e051608. doi:10.1136/ bmjopen-2021-051608

- Prepublication history and additional supplemental material for this paper are available online. To view these files, please visit the journal online (http://dx.doi.org/10.1136/ bmjopen-2021-051608)

$\mathrm{X}-\mathrm{MH}$ and $\mathrm{F}-\mathrm{ZY}$ are joint first authors.

Received 24 March 2021 Accepted 14 January 2022

Check for updates

(c) Author(s) (or their employer(s)) 2022. Re-use permitted under CC BY-NC. No commercial re-use. See rights and permissions. Published by BMJ.

For numbered affiliations see end of article.

Correspondence to

Dr Jia-Kuo Yu;

yujiakuo@126.com

\section{ABSTRACT}

Objective Are physical therapy or orthopaedic equipment efficacious in reducing the biomechanical risk factors in people with tibiofemoral osteoarthritis $(0 \mathrm{~A})$ ? Is there a better therapeutic intervention than others to improve these outcomes?

Design Systematic review with network meta-analysis (NMA) of randomised trials

Data sources PubMed, Web of Science, Cochrane Library, Embase and MEDLINE were searched through January 2021.

Eligibility criteria for selecting studies We included randomised controlled trials exploring the benefits of using physical therapy or orthopaedic equipment in reducing the biomechanical risk factors which included knee adduction moment (KAM) and knee adduction angular impulse (KAAl) in individuals with tibiofemoral $O A$.

Data extraction and synthesis Two authors extracted data independently and assessed risk of bias. We conducted an NMA to compare multiple interventions, including both direct and indirect evidences. Heterogeneity was assessed (sensitivity analysis) and quantified $\left(\mathrm{I}^{2}\right.$ statistic). Grading of Recommendations Assessment, Development and Evaluation assessed the certainty of the evidence.

Results Eighteen randomised controlled trials, including 944 participants, met the inclusion criteria, of which 14 trials could be included in the NMA. Based on the collective probability of being the overall best therapy for reducing the first peak KAM, lateral wedge insoles (LWI) plus knee brace was closely followed by gait retraining, and knee brace only. Although no significant difference was observed among the eight interventions, variable-stiffness shoes and neuromuscular exercise exhibited an increase in the first peak KAM compared with the control condition group. And based on the collective probability of being the overall best therapy for reducing KAAl, gait retraining was followed by LWI only, and lower limb exercise.

Conclusion The results of our study support the use of LWI plus knee brace for reducing the first peak KAM. Gait retraining did not rank highest but it influenced both KAM
Strengths and limitations of this study

- The Bayesian method provided the probability estimates regarding the relative efficacy of specific interventions, even though standard methods found no significant differences among them.

$>$ Physical therapies and orthopaedic equipment are complex interventions with a small number of trials comparing the different types of interventions.

- Besides knee adduction moment and knee adduction angular impulse, we were unable to include other biomechanical risk factors, such as the external knee flexion moment to joint load, because the number of these studies was not enough to form a complete network meta-analysis (NMA).

- Heterogeneity in NMA may reduce the validity of the results.

and KAAI and therefore it was the most recommended therapy for reducing the biomechanical risk factors.

\section{INTRODUCTION}

Knee osteoarthritis (KOA), a chronic progressive disease, affects approximately $3.8 \%$ of people worldwide and frequently occurs in the middle-aged and the elderly population. ${ }^{1}$ The main clinical manifestation of KOA is knee pain and is often accompanied by radiographic degeneration of the intra-articular cartilage associated with hypertrophic bone changes. $^{2}$ Furthermore, the KOA development often leads to knee stiffness, joint locking and instability, along with functional loss. Though it is not fatal, the persistent pain and movement restrictions associated with this condition negatively impact the physical and mental health of the patients, thus, reducing their quality of life. ${ }^{3}$ 
These pathological changes in knee joints are a cumulative result of various biomechanical imbalances leading to the progression of the disease and are now believed to be associated with malalignment of the lower limb. ${ }^{4}$ Tibiofemoral OA most commonly occurs in the medial compartment, since several studies have stated that patellofemoral compartment is as prevalent as medial tibiofemoral joint. $^{5} 6$ The external knee adduction moment (KAM) results from the unequal distribution of the transmitted load on both sides in the normal gait of humans. It is defined as the cross product of the ground reaction force and the distance between the knee joint and the force line. ${ }^{7}$ Individuals with obesity, ${ }^{8}$ meniscal lesions, ${ }^{9}$ occupational loads ${ }^{10}$ or other associated risk factors tend to have a frontal plane knee malalignment, which alters the normal force line and forces the medial knee joint to bear more load and thus, leads to increased KAM. ${ }^{11} 12$ The accumulation effect of the moment is determined by calculating the integral of the moment to time, which is also called knee adduction angular impulse (KAAI). It reflects the change in knee joint rotation state during a stance period of gait. ${ }^{13}$ Previous studies have revealed a strong correlation between the peak levels of KAM and KAAI and the severity and progression of the disease, which was reflected and calculated by the loss of medial tibial cartilage. ${ }^{1415}$ Both these biomechanical parameters (KAM and KAAI) are commonly used to evaluate the medial knee load and predict the long-term structural deterioration of the knee.

Recent advancements in healthcare have resulted in the development of several protocols for the intervention and treatment of KOA. Patients with KOA are primarily recommended physical therapy or orthopaedic equipment with the intention of correcting the deviated force line and delaying the progressive pathological damage inside the knee joint. ${ }^{7}$ Some other modalities, such as ultrasound and Taiji programmes, primarily focus on relieving the pain, and therefore, this might improve the biomechanical state of the knee joint. ${ }^{16}{ }^{17}$ The physical therapy mainly includes muscular strengthening, exercise therapy, electric stimulation therapy, extracorporeal shockwave therapy and gait modification, while orthopaedic equipment mainly incorporates customised shoes/footwear, wedged insoles and knee braces.

Several literary insights have shown the positive impact of physical therapy or orthopaedic equipment in patients with KOA. ${ }^{131819}$ The strengthening of related lower limb muscles, which play a vital role in disease progression, are known to reduce instability and abnormal stresses across the joint. ${ }^{20}{ }^{21}$ Another study displayed a lower knee joint loading rate in patients with stronger quadriceps and hamstrings. ${ }^{22}$ Additionally, gait training presents a viable way for correcting the patients' underlying gait pattern, thus, further reducing their knee load and pain. ${ }^{23} 24$ Furthermore, various kinds of orthotic devices have been introduced for the treatment of KOA. The clinical use of lateral wedge insoles (LWI) has gained immense popularity since its origin in $1987 .{ }^{2526}$ The insoles tends to shift the lateral part of the foot more than the medial part by a slope that increases the valgus tendency of lower extremities. The centre of the ground reaction force is shifted laterally, which induces a reduction in force lever arm length and magnitude. ${ }^{27}$ Also, the valgus knee brace is a commonly used device. It applies an external valgus force around the knee joint to reduce the medial knee load.

In the past, several systematic reviews and meta-analyses have been published featuring the medical effects of a single KOA treatment. However, only a few of them have focused on multifaceted interventions. Also, only a few reviews have reported the effects on biomechanical parameters. The mechanical changes in the body were not sufficiently investigated. Current reviews on KAM and KAAI have also not compared these changes. Thus, a network meta-analysis (NMA) was performed to appraise the benefits of physical treatments or orthopaedic equipment in reducing biomechanical risk factors in patients with KOA.

Therefore, the research questions for this systematic review were:

1. Are physical therapies or orthopaedic equipment efficacious in reducing the biomechanical risk factors in people with KOA?

2. Is there a better therapeutic intervention than others to improve these outcomes?

\section{METHODS}

All pooled analyses were derived from previous studies and, therefore, did not require ethical approval and informed consent.

\section{Identification and selection of studies}

The following databases were searched for listed randomised controlled trials that were published before January 2021: PubMed, Web of Science, Cochrane Library, Embase and MEDLINE. These studies explored the benefits of using physical therapy or orthopaedic equipment in reducing the biomechanical risk factors including KAM and KAAI in patients with tibiofemoral OA. The search was not restricted by date, publication type or status (see online supplemental appendix 1). Additionally, we performed manual analyses of the published references regarding the use of physical therapy or orthopaedic equipment for treating KOA.

The eligibility of searched publications was independently reviewed by X-MH and Z-XY following the Cochrane manual directives. ${ }^{28}$ Any additional inconsistencies were resolved either by deliberation or by a senior expert $(\mathrm{YH})$. First, the study titles and abstracts, published in English literature, were screened. Next, the complete articles were reviewed against the directed criteria described in box 1 .

Eligible comparison subjects, including standard/ conventional care or waiting list control (analgesic advice and education), were defined as 'control condition'. Control condition also included placebo intervention, no 


\section{Box 1 Inclusion criteria}

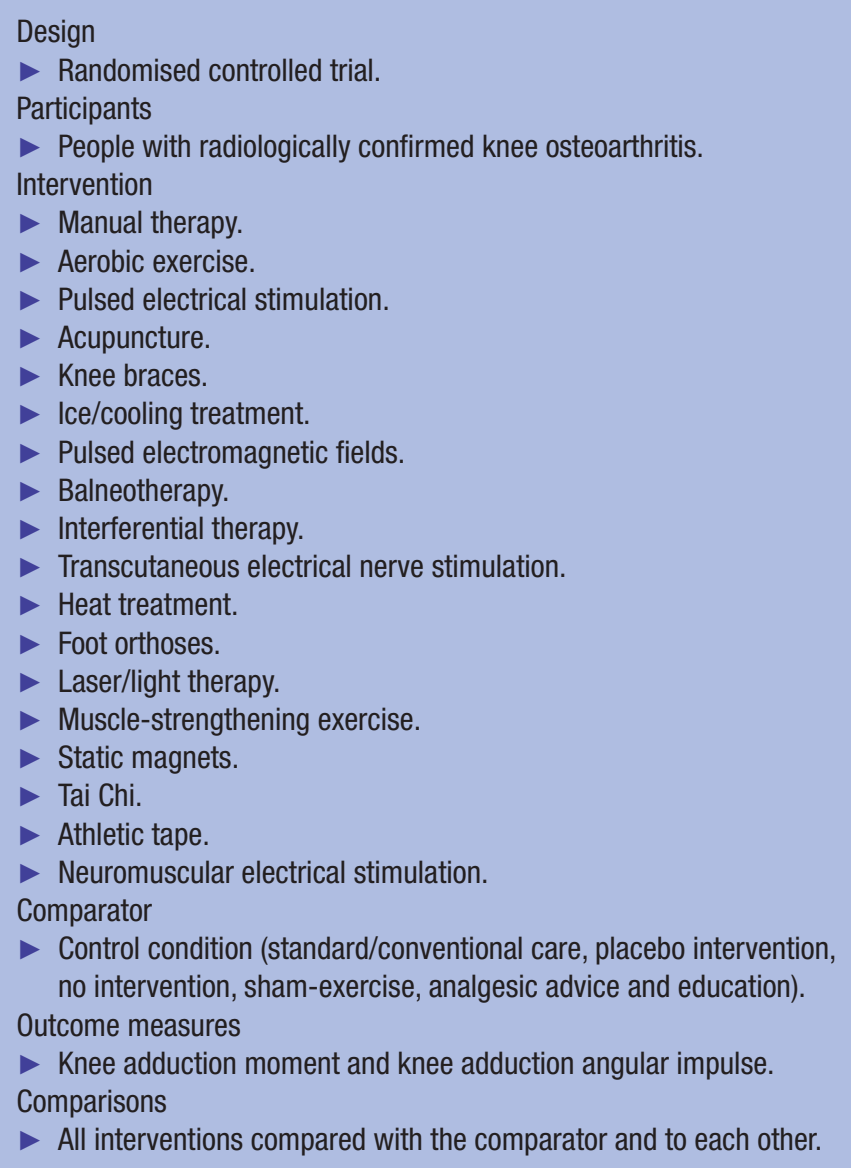

intervention and sham-exercise. This NMA defined lower limb exercise as the simultaneous exercise of multiple muscle groups that included hip abductors, quadriceps and hamstrings. Since our research needed to maintain clinical and statistical homogeneity and focus on the residual biomechanical effects after the intervention, only those articles were selected whose measurements were strictly obtained under the condition of going barefoot.

The exclusion criteria included: (1) studies that were not consistent with the eligibility criteria; (2) experimental peer-reviewed studies; (3) studies that included participants who had received surgical treatment in the past; and (4) studies that did not report KAM or KAAI.

\section{Data collection and quality assessment}

KAM and KAAI were the preferred biomechanical measures used in this meta-analysis. The biomechanical outcomes of the studies included in the Bayesian NMA were measured on flat ground or treadmills. Additionally, the number of trials focusing on the second peak of KAM was insufficient to conduct an independent NMA.

The data were extracted independently by two authors (X-MH and Z-XY) and were cross-checked. A predefined information sheet was used for data extraction, which included the details of the first author (name), country, the year of publication, population characteristics, intervention and the time points. The authors of the original study were contacted in the cases requiring more data.

\section{Assessment of characteristics of studies \\ Risk of bias}

This NMA used the Cochrane Risk of Bias 2 to assess the risk of bias in randomised controlled trials using the following evaluation indicators: randomisation process, deviations from the intended interventions, missing outcome data, outcome measurement and selection of the reported results. ${ }^{21}$ The judgement of the bias risk of this item was presented as 'low', 'high' and 'some concerns'. Two authors independently evaluated the risk of bias in all the included studies. The authors discussed or referred to the opinion of a senior author to resolve any disagreements. Additionally, the certainty of the evidence was also evaluated, which contributed to network estimates of the main outcomes with the Grading of Recommendations Assessment, Development and Evaluation (GRADE) framework. ${ }^{29}$

\section{Intervention}

In order to describe the experimental interventions, the following information was extracted: the training method with further relevant details, the details and characteristics of orthopaedic equipment and the frequency as well as the total duration of training or wearing.

\section{Outcome measures}

Baseline biomechanical risk factors were extracted from the walking trials without any orthopaedic equipment before the intervention, while post-intervention biomechanical risk factors were extracted from walking trials that incorporated orthopaedic equipment. Biomechanical risk factors included in the study were the first peak KAM, the second peak KAM and KAAI. KAM was normalised as \%body weight times height, with conversion to $\mathrm{Nm} / \mathrm{kg}$ wherever necessary. KAAI was designated as the moment accumulation rate, which was determined by calculating the integral of the moment to time.

\section{Statistical analysis}

An NMA was carried out for comparing multiple interventions, including both direct (direct comparison of treatment modalities) and indirect evidence (indirect comparison of various treatments with a common control), maintaining randomisation in each independent study. ${ }^{30-32}$ Interventions, as well as different demographic characteristics were either consistent or comparable in all included studies, ${ }^{303-37}$ while those studies were excluded that reported immediate treatment effects.

Due to different units, the continuous data used the standard mean difference as the statistical indicator of the effect, and the frequentist $95 \%$ CI of each effect was calculated. Additionally, the $\mathrm{I}^{2}$ statistic was used to analyse the overall heterogeneity of the two-arm study and the network. The fixed-effect model was suggested to be used in cases of the absence of statistical heterogeneity $(p>0.05$, 
$\mathrm{I}^{2}<50 \%$ ); however, given the heterogeneity among the studies, a random-effects model for meta-analysis was used. $^{38}$ A sensitivity analysis (see online supplemental appendix 2, eFigures 1 and 2) was conducted by omitting one study and investigating the influence of the single study on the overall pooled estimate to evaluate the source of heterogeneity. The node-split model was used for evaluating the testing consistency (see online supplemental appendix 3, eFigure 3). If $\mathrm{p}>0.05$, then the consistency model was used for analysis; otherwise, the inconsistency model was used..$^{39}$ Normal likelihood distributions were assumed, non-informative prior distributions were set and three Markov chains were run simultaneously. Since the number of update iterations was 50000 , a total of 5000 simulations were used for annealing, and the subsequent 45000 iterations were examined. The mean rank and surface under the cumulative ranking curve (SUCRA) were used for reporting the probability values. A SUCRA value of $100 \%$ was considered best, whereas $0 \%$ indicated the worst treatment. ${ }^{40}$ Besides, a conventional metaanalysis was also carried out (see online supplemental appendix 4, eFigure 4A-C). Comparison-adjusted funnel plots were prepared that represented different comparisons with different colours.

The data from the eligible studies were combined using the Review Manager (RevMan) software V.5.3. The contribution of the effect sizes was dependent on the sample size and their estimation accuracy. The Bayesian analyses were carried out using WinBUGS V.1.4.3. Stata (StataCorp. 2015. Stata Statistical Software: Release 15) was employed to conduct the frequentist NMA.

\section{Patient and public involvement}

No patients were directly involved in the development of the study question, selection of the outcome measures, design and implementation of the study or explanation of the results.

\section{RESULTS}

\section{Flow of studies through the review}

A comprehensive investigation of databases retrieved 4919 citations. After screening articles by title and abstract, and deleting duplicate articles, we identified 526 studies that might meet the criteria for inclusion, and then we searched and evaluated their full text. Figure 1 presents the study selection flow chart. Eighteen randomised controlled trials, including 944 participants, met the inclusion criteria. ${ }^{231-57}$ Since the present NMA only considered trials comparing the nine treatments with control condition or each other (see online supplemental appendix 5, eFigure 5A,B), only 14 trials (792 participants) were included. Furthermore, four trials were excluded from the NMA considering their excessive heterogeneity and inability to form NMA with other studies. ${ }^{54-57}$

\section{Characteristics of included studies}

All studies included tibiofemoral OA cases, which were radiologically confirmed. Although most interventions

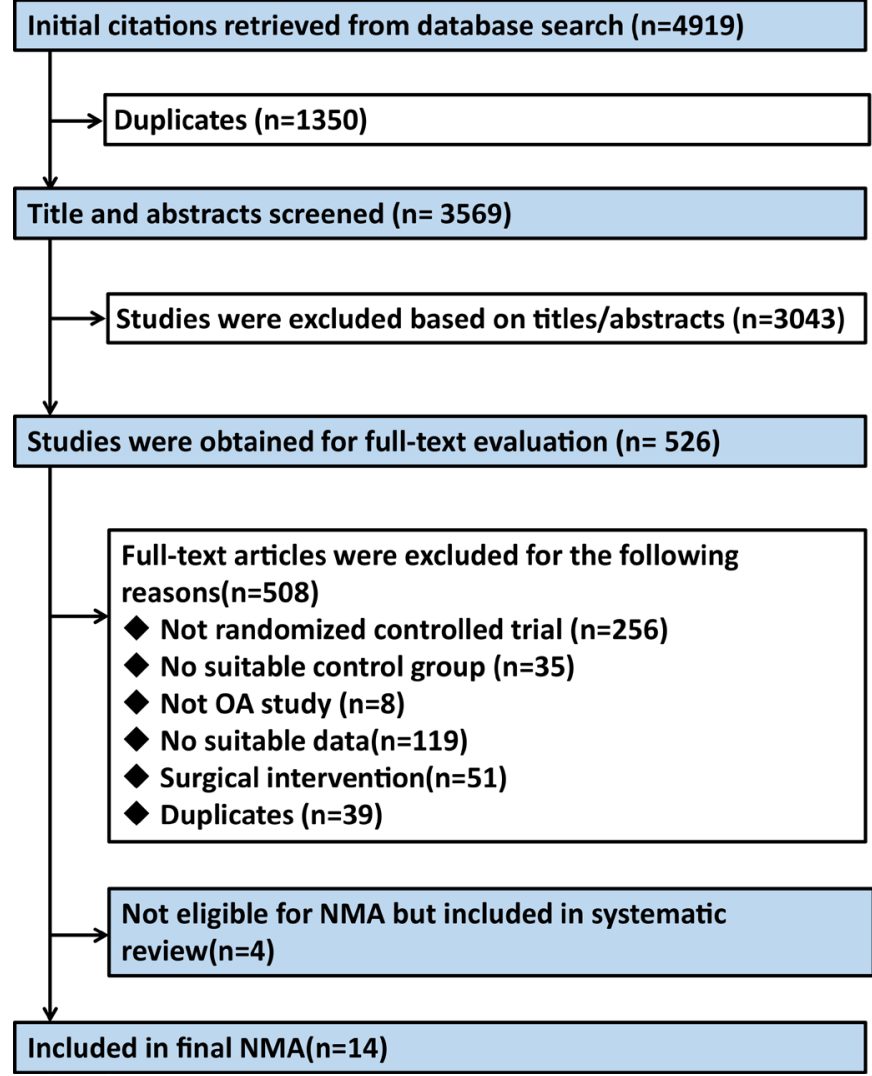

Figure 1 Flow chart of the study selection. NMA, network meta-analysis; OA, osteoarthritis.

were administered over an 8-13 week period, the treatment duration ranged from 2 weeks to 12 months. The number of exercises varied from 2 to 5 times per week, depending on the initial preparation. ${ }^{4344649}$ Both gait training studies used the faded feedback paradigm, which meant gradual removal of the real-time biofeedback. ${ }^{23} 48$ As NMA included 14 studies, 9 were classified as Kellgren/Lawrence grade 2 and above. All studies reported either the values for body mass index (BMI) or height and weight, while the studies recruiting a general population classified the mean BMI as overweight or obese. Additionally, one NMA study had a randomised crossover design. ${ }^{50}$ After consulting a reference manual along with a professional statistician, the mean and SD of the experimental and the control groups were analysed in this NMA. ${ }^{28}$ Tables 1 and 2 summarise the characteristics of the included studies and their participants.

\section{KAM}

According to the collective probability of being the overall best therapy for reducing the first peak KAM, LWI plus knee brace $(93.4 \%)$ was closely followed by gait retraining $(85.7 \%)$, and knee brace only $(79.3 \%)$ (figure 2). A study reported that the VER-brace (unloader brace with valgus and external rotation functions) offers additional advantages on first peak KAM compared with V3P-brace (three-point bending system valgus knee brace) and ACLbrace (functional medial-lateral stabilisation brace used after ligament injuries). ${ }^{54}$ No first peak KAM reduction 

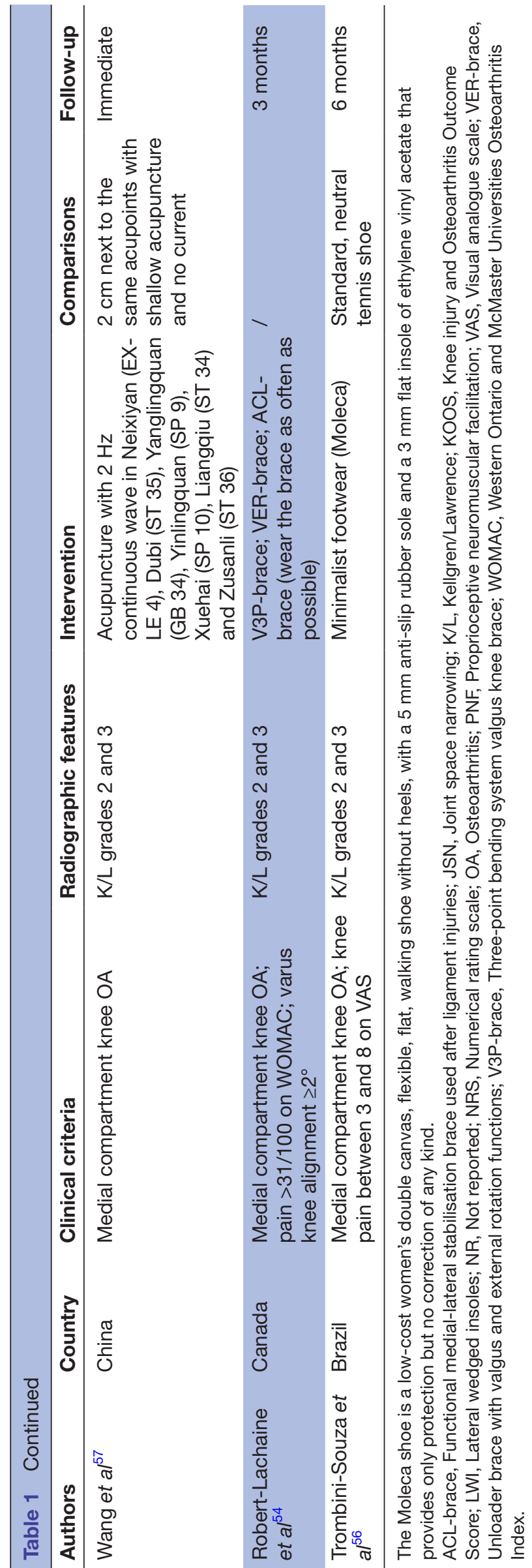

was observed between proprioceptive neuromuscular facilitation group and controls, ${ }^{55}$ and the result of the study of minimal footwear was the same. ${ }^{56}$ On the other hand, after the electroacupuncture treatment, compared with the control group, the second peak KAM significantly increased immediately when the patient ascended stairs. ${ }^{57}$ Table 3 shows the NMA results of a comparative analysis of the reduction of the first peak KAM. We found no differences in most of the treatment modalities; however, variable-stiffness shoes showed a statistically significant increase in the first peak KAM over the rest of the included interventions. Neuromuscular exercise was better than variable-stiffness shoes, but was still inferior to most other interventions. At the same time, lateral wedge insole plus knee brace and gait retraining performed relatively well in reducing the first peak KAM compared with control condition and other treatments.

\section{KAAI}

Based on the collective probability of being the overall best therapy for reducing KAAI, gait retraining (90.7\%) was followed by LWI only $(74.1 \%)$, and lower limb exercise $(53.8 \%$ ) (figure 3). KAAI was reported in 10 studies. ${ }^{42-44} 47-50535456$ After wearing the three kinds of brace separately, the KAAI measured without brace did not decrease significantly, and there was no significant difference between the groups. ${ }^{54}$ Table 3 shows the NMA results of the reduction of KAAI. Most treatments were not statistically different from each other, consistent with the results of the first peak KAM. Only gait retraining had a statistical reduction compared with control condition. The aggregated results suggested that gait retraining is efficacious in reducing the KAAI, while neuromuscular exercise increased the KAAI compared with gait retraining and knee brace.

\section{Heterogeneity}

We removed a study which had a short follow-up time and might cause heterogeneity, ${ }^{50}$ and performed another NMA. There was no difference between the results of the reanalysis and the current ranking (see online supplemental appendix 2, eFigures 1 and 2).

\section{GRADE assessment}

According to the GRADE framework (see online supplemental appendix 6), the quality of most comparisons was assessed as low or very low. Only neuromuscular exercise compared with control condition, neuromuscular exercise compared with LWI, neuromuscular exercise compared with knee brace and neuromuscular exercise compared with LWI plus knee brace were evaluated as a moderate-grade comparison.

\section{Risk of bias}

Figure 4 depicts a summary of the risk-of-bias scores for the included randomised controlled trials (RCTs) in this analysis. Nine studies presented a clear description of generating a randomisation sequence. ${ }^{43-47} 49525657$ The study by Hinman et al was the only double-blinded study, 


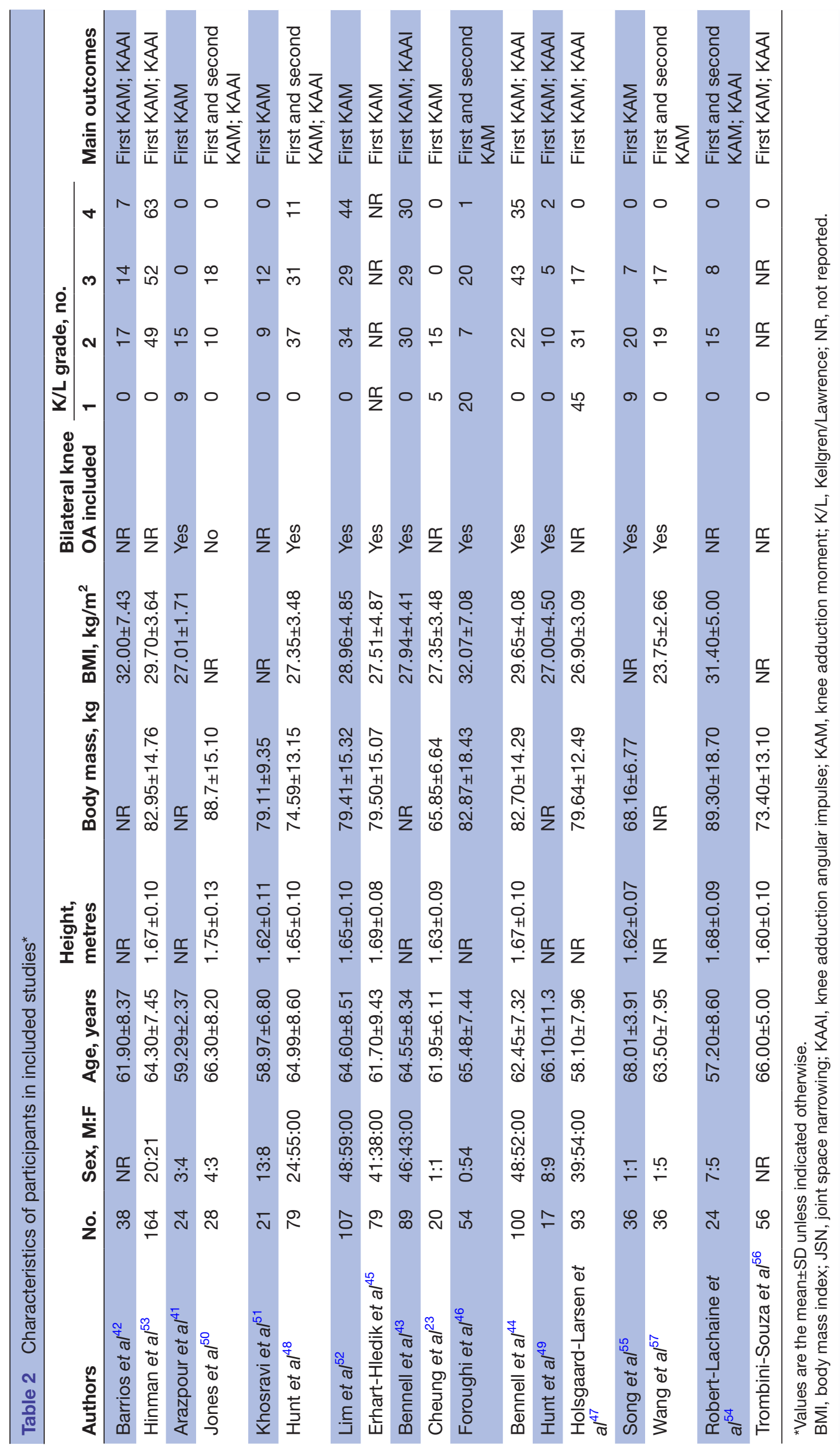

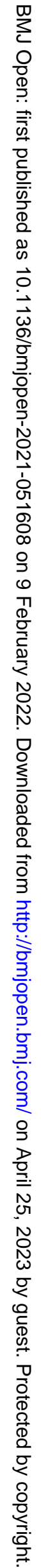




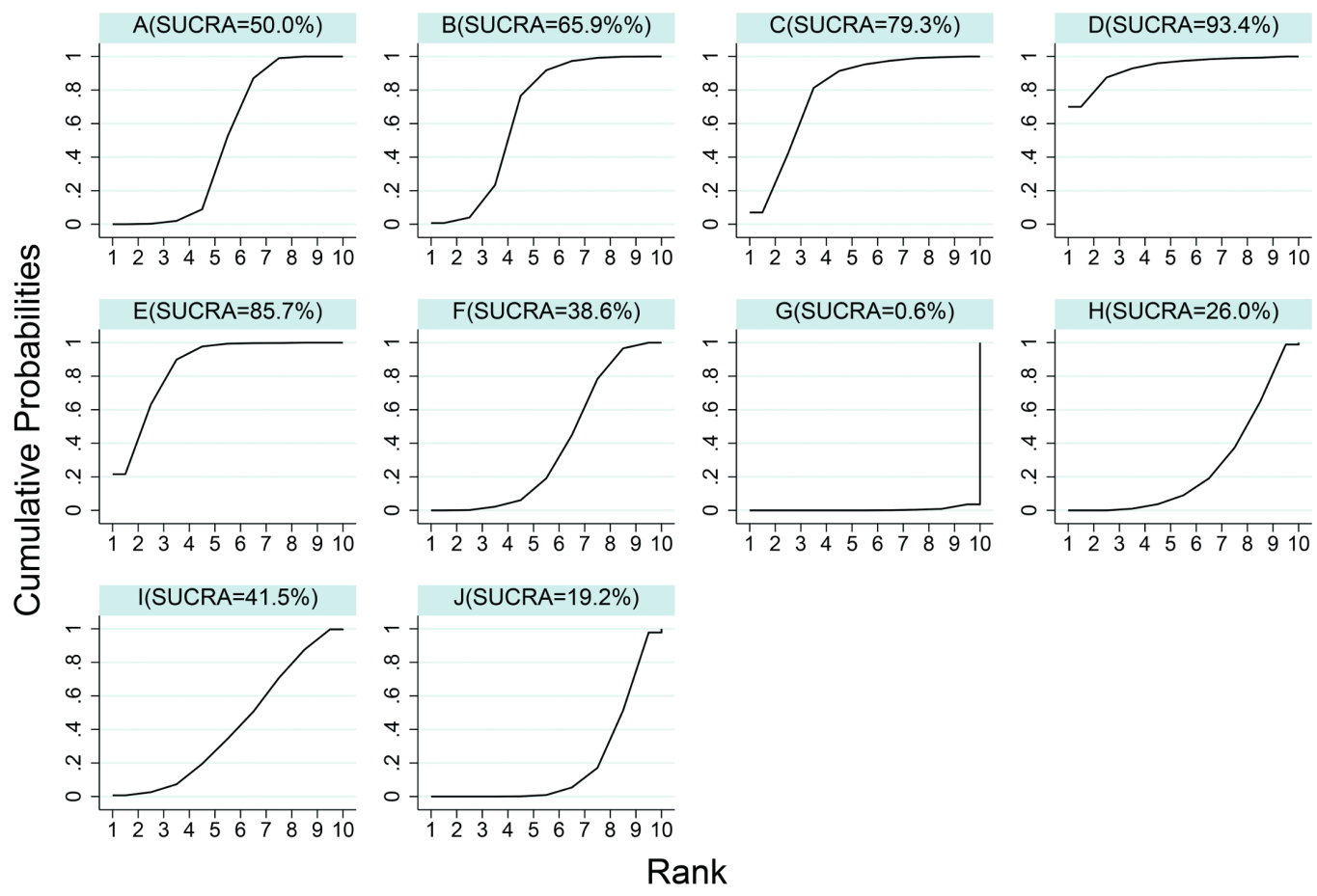

Graphs by Treatment

Figure 2 Rankings for effects on first peak knee adduction moment. The graph displays the distribution of probabilities for each treatment. The $X$-axis represents the possible rank of each treatment (from the best to worst according to the outcomes), $\mathrm{Y}$-axis represents the cumulative probability for each treatment to be the best option, among the best two options, among the best three options and so on. A, control condition; B, lateral wedge insole; C, knee brace; D, lateral wedge insole + knee brace; E, gait retraining; F, quadriceps strengthening; G, variable-stiffness shoe; H, hip strengthening; I, lower limb exercise; J, neuromuscular exercise; SUCRA, surface under the cumulative ranking curve.

while other studies were either single-blinded or did not clearly describe their blind design. All trials provided follow-up data on their outcomes. Six studies did not report the patient number or the reason for lost visits due to the length of follow-up. ${ }^{2344-465052}$ All studies were included in the synthesis evaluation. The comparisonadjusted funnel plots were symmetrically distributed based on a visual inspection, which suggested the absence of small-sample effects for our study outcomes (see online supplemental appendix 7, eFigure 6A,B) .

\section{DISCUSSION}

Our study results did not show any significant difference regarding the relative efficacy of intervention among different types of physical therapies or orthopaedic equipment. This lack of difference might be attributed to the fact that the number of studies for several pairwise comparisons was small. However, some of these therapies were still worth recommending. Due to a small number of studies studying the outcome of the KAAI, we found gait retraining to be the relatively more convincing intervention as it could simultaneously reduce the values for KAM and KAAI values based on cumulative ranking and relative effect estimates. Due to the lack of significant differences among the interventions, the cumulative ranking obtained by the NMA could not be conclusively accepted. For example, gait retraining, which was employed as the foremost intervention $(90.7 \%)$ for KAAI reduction, was only superior to the neuromuscular exercise interventions.

This study had several strengths and limitations. This NMA is the first report on the effects of physical therapy or orthopaedic equipment on the parameters of knee load (KAM and KAAI). Since physical therapies and orthopaedic equipment are complex interventions with a small number of trials comparing the different types of interventions, NMA was deemed as the most relevant form of analysis. The results of this meta-analysis could be more useful for the decision-makers and primary service providers for choosing wisely among the various available options, as compared with the multiple separate pairwise meta-analyses. ${ }^{58}$ Additionally, this NMA conducted each comparison distinctly with both direct and indirect statistical effects, deriving statistical power from all included data. ${ }^{58}$ Also, the Bayesian method provided the probability estimates regarding the relative efficacy of specific interventions, even though the standard methods described the absence of a significant difference between them. Furthermore, alternative rankings (second, third best, etc) were calculated to provide overall feasibility due to 


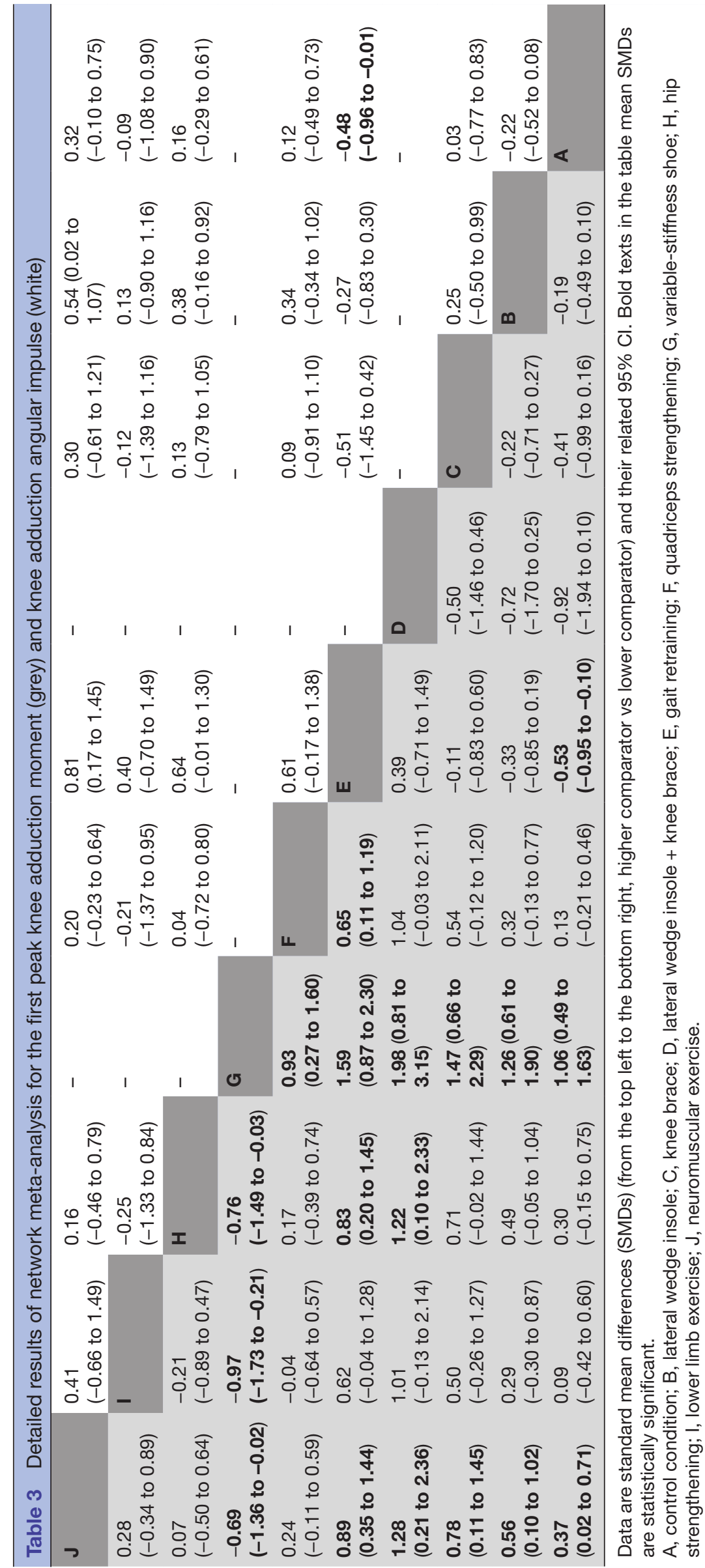




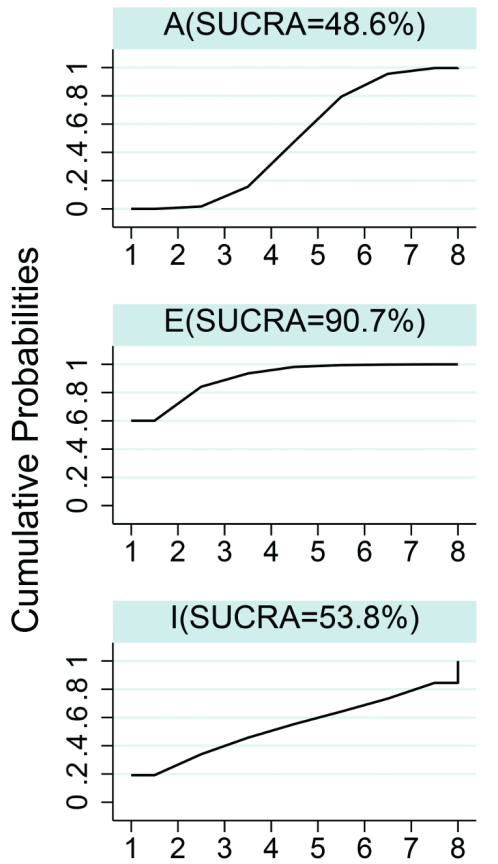

Graphs by Treatment
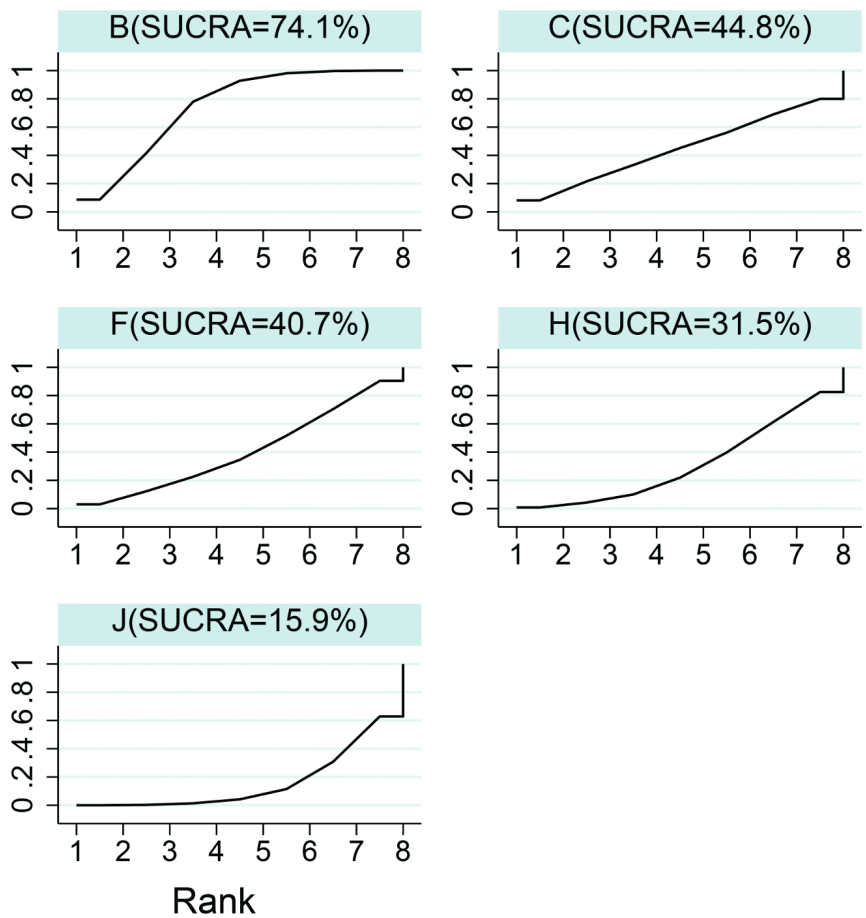

Figure 3 Rankings for effects on knee adduction angular impulse. The graph displays the distribution of probabilities for each treatment. The $\mathrm{X}$-axis represents the possible rank of each treatment (from the best to worst according to the outcomes), $\mathrm{Y}$-axis represents the cumulative probability for each treatment to be the best option, among the best two options, among the best three options and so on. A, control condition; B, lateral wedge insole; C, knee brace; E, gait retraining; F, quadriceps strengthening; $\mathrm{H}$, hip strengthening; I, lower limb exercise; J, neuromuscular exercise; SUCRA, surface under the cumulative ranking curve.

unavailability of the best-suited interventions, more expensive therapies or contraindications in some cases. As with most meta-analyses based on non-surgical therapies for osteoarthritis, one of the limitations of this NMA was the inclusion of trials that had variable periods of follow-up, which might have introduced heterogeneity into the study analyses. The Cochrane handbook recommends several methods for analysing and comparing trials with multiple durations of follow-up, as recommended by the Cochrane handbook, such as performing individual patient data meta-analysis and a precise evaluation at a particular time point. However, newer approaches are now being developed that would include all the time points in an NMA. ${ }^{28}$ Our study was unable to evaluate the influence of population characteristics (such as mean age, the severity of osteoarthritis), as the number of the included studies was not large enough. ${ }^{59-61}$ Additionally, other parameters, such as the external knee flexion moment to joint load, should have been studied in detail. However, due to lesser available literature, our study was unable to include them. Finally, standard/conventional care, placebo intervention, no intervention, sham-exercise, analgesic advice and education were all considered as the same parameter in defining the 'control condition'. Therefore, the relative rankings in our study might not represent the true factual rankings as compared with actual standard care due to lack of consideration of bias introduced by heterogeneity and lack of blinding.

A previous review reported that LWIs were able to reduce the KAM at the baseline ${ }^{13}$; however, the effect was no longer observed after a specific period. Another study displayed that a month wear-in period was the longest study time in which no reduction in biochemical risk factors was observed despite continued wear. ${ }^{18}$ Besides, several other systematic reviews stated that exercise and gait retraining could further reduce pain and improve motor functioning in people with KOA. ${ }^{62-64}$ There is a high probability that any clinical changes occurring in previous studies might be due to increased physical activity levels, and not owing to the altered loading environment within the knee joint. Furthermore, another study revealed that an increase in the amount of reduction in peak KAM in LWIs plus knee brace group was observed after 4 weeks. ${ }^{65}$ In our NMA, we focused on the studies of non-immediate effect, removed the research with a follow-up time of less than 1 month in the sensitivity analysis and made the final rank. Our results showed that only gait training produces a significant reduction in KAM and KAAI when compared with control condition, indicating that the biomechanical reduction effect of orthopaedic equipment cannot be maintained for a long time when they are donned. It was evident that an 


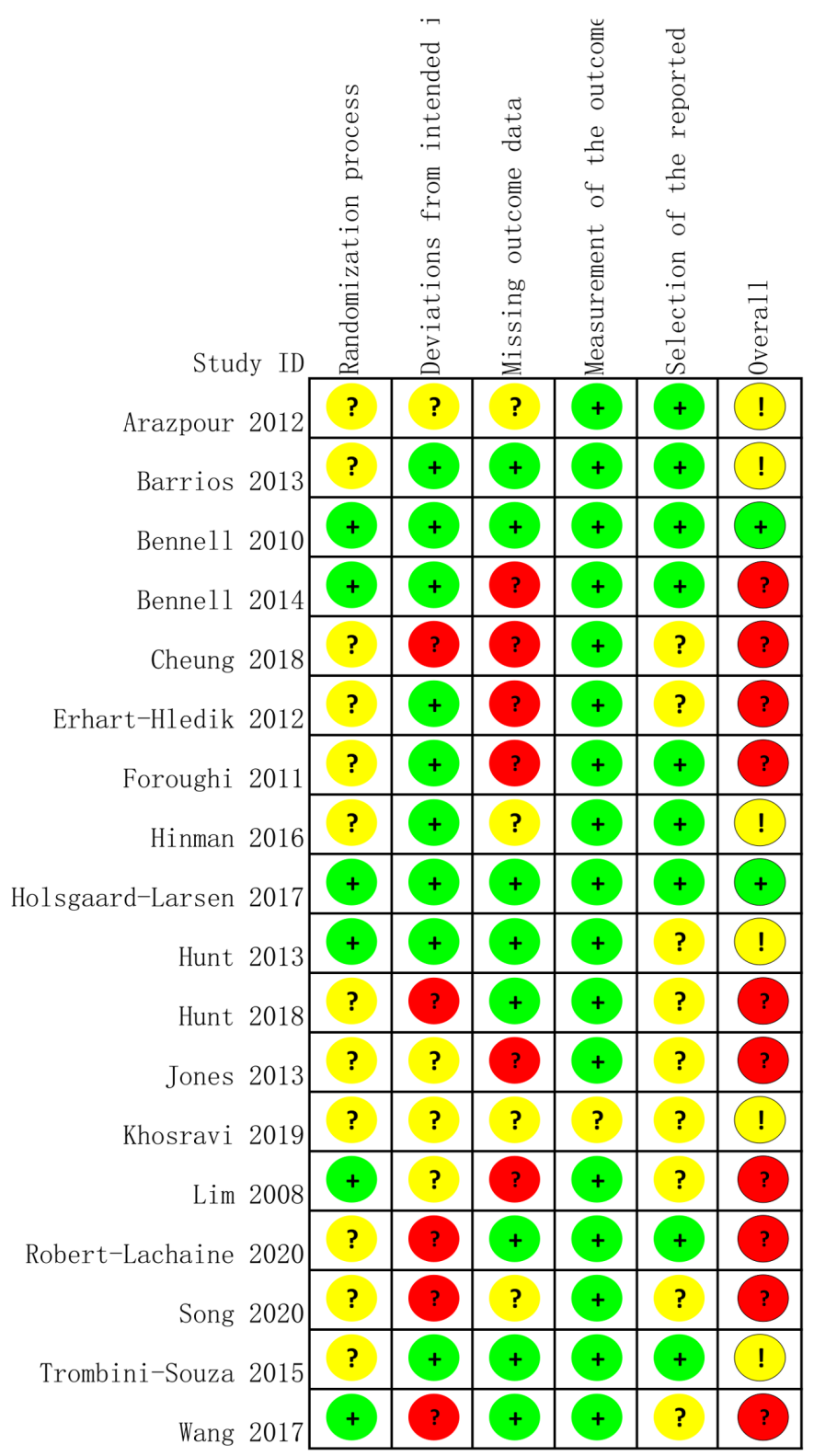

Figure 4 Risk of bias summary.

extension of the treatment time led to a decrease in the biomechanical reduction effect, which might be due to the gradual deformation of the orthopaedic equipment that renders them ineffective, despite being made from high-density materials.

On the other hand, various physical therapies and orthopaedic equipment also should be considered for relieving patients' pain, which has been the focus of several past reviews. As an important gait parameter, the joint pain can affect the kinetics and kinematics of walking. ${ }^{19}$ A meta-analysis reported that exercise therapy had a positive impact on knee pain and kinematic function, though this relief of pain subsided with time. After proper initiation, the efficiency of physical exercise over placebo reached a maximum level at 2 months. ${ }^{66}$

Cumulative loading is another significant parameter regarding knee load exposure in OA. ${ }^{67}$ KAAI has been proposed as another indicator for evaluating the duration and intensity of KOA load, despite the association between KAM and disease progression. According to a study lasting for a year, the loss of medial tibiofemoral cartilage was not directly linked to KAM but was promptly related to KAAI. ${ }^{14}$ Although the effect of physical therapy or orthopaedic equipment on KAM are short-lived, it might have a huge cumulative effect on the knee during the early stages of treatment and should be considered while interpreting our NMA results.

Our study results are both scientifically and clinically instructive. Despite a majority of therapies displaying a null statistical KAM and KAAI reduction, the clinical usage of these treatment modalities could significantly improve the presenting symptoms and physical activity level without increasing the biomechanical magnitude; thus, improving the quality of life of patients with KOA. Although the results of this study suggested that wearing variable-stiffness shoes is not preferable for long-term KAM reduction, our current study explained that variable-stiffness shoes displayed a major advantage in reducing KAM for patients with increasing walking speed. ${ }^{68}$ At the same time, variable-stiffness shoes had relatively less discomfort than equipment such as LWI. Since the studies included in this NMA mainly involves patients with medial KOA, the consolidated results would be more useful for such patients.

On the other hand, a previous study reported that an increase in KAAI can explain the significant variation in the uCTX-II levels as well as the uCTX-II:sCPII ratio in patients with medial tibiofemoral KOA after controlling additional variables. ${ }^{49}$ It was evident that appropriate intervention in the biomechanical structure of the knee joint in patients with KOA exert a potential beneficial role on cartilage structure. Maleki et al reported that adopting a modified gait for reducing the KAM can decrease the pain in the medial compartment in KOA more than walking alone ${ }^{69}$ which suggests that the KAM and KAAI of patients undergoing non-surgical approaches could be restricted to reduce pain and improve the joint function. More research is further needed to promptly illustrate the impact of changes in knee biomechanics on the prognosis of such patients.

Additionally, some other therapies have also been reported, such as Taiji, ultrasound, acoustic exercises. However, due to the lack of RCT study design or the report of their biomechanical outcomes, these therapies were not included in our review. Therefore, further studies would require more research articles in these areas for exploring the impact of various non-surgical therapies on patients with OA. After accumulating evidence regarding the role of non-surgical therapy in KOA, another similar NMA to understand the relative effectiveness of various treatment in the relevant patients.

\section{CONCLUSION}

To conclude, this NMA provides valuable insights regarding the KAM and KAAI alterations in patients with 
OA after the usage of physical therapy or orthopaedic equipment. After integrating cumulative ranking and relative effect estimates, LWI plus knee brace was the highest-ranking intervention despite an absence of statistical significance. Although gait retraining did not score a higher rank, it remarkably influenced both KAM and KAAI values and, therefore, was the most recommended therapy for reducing the biomechanical risk factors. On the contrary, variable-stiffness shoe and neuromuscular exercise should be used with caution in clinical practice. Taken together, these findings suggest that clinicians should carefully consider all appropriate treatment modalities when treating patients with OA.

\section{Author affiliations}

${ }^{1}$ Department of Sports Medicine, Peking University Third Hospital, Institute of Sports Medicine of Peking University, Beijing Key Laboratory of Sports Injuries, Beijing,

China

${ }^{2}$ Plastic Surgery Hospital, Chinese Academy of Medical Sciences and Peking Union Medical College, Beijing, China

${ }^{3}$ Department of Orthopedic, Peking University Third Hospital, Beijing, China

Contributors $\mathrm{X}-\mathrm{MH}$ and F-ZY conceived of the study, and participated in its design and coordination and helped to draft the manuscript; Z-XY, YH and Y-RC contributed significantly to analysis and manuscript preparation; J-KY and LL helped perform the analysis with constructive discussions and revised it critically for important intellectual content. J-KY is responsible for the overall content as the guarantor.

Funding This work was supported by the National Key R\&D Program of China (No.2017YFB1303000) and the Construction of a Basic Public Service Platform for Industrial Technology in the Field of Advanced Medical Equipment (0714-EMTC-02-00897).

Competing interests None declared.

Patient consent for publication Not applicable.

Ethics approval This study does not involve human participants.

Provenance and peer review Not commissioned; externally peer reviewed.

Data availability statement № data are available.

Supplemental material This content has been supplied by the author(s). It has not been vetted by BMJ Publishing Group Limited (BMJ) and may not have been peer-reviewed. Any opinions or recommendations discussed are solely those of the author(s) and are not endorsed by BMJ. BMJ disclaims all liability and responsibility arising from any reliance placed on the content. Where the content includes any translated material, BMJ does not warrant the accuracy and reliability of the translations (including but not limited to local regulations, clinical guidelines, terminology, drug names and drug dosages), and is not responsible for any error and/or omissions arising from translation and adaptation or otherwise.

Open access This is an open access article distributed in accordance with the Creative Commons Attribution Non Commercial (CC BY-NC 4.0) license, which permits others to distribute, remix, adapt, build upon this work non-commercially, and license their derivative works on different terms, provided the original work is properly cited, appropriate credit is given, any changes made indicated, and the use is non-commercial. See: http://creativecommons.org/licenses/by-nc/4.0/.

ORCID iD

Xi-Meng Huang http://orcid.org/0000-0002-8420-5649

\section{REFERENCES}

1 Safiri S, Kolahi A-A, Smith E, et al. Global, regional and national burden of osteoarthritis 1990-2017: a systematic analysis of the global burden of disease study 2017. Ann Rheum Dis 2020;79:819-28.

2 Turpin KM, De Vincenzo A, Apps AM, et al. Biomechanical and clinical outcomes with shock-absorbing insoles in patients with knee osteoarthritis: immediate effects and changes after 1 month of wear. Arch Phys Med Rehabil 2012;93:503-8.
3 Neogi T. The epidemiology and impact of pain in osteoarthritis. Osteoarthritis Cartilage 2013;21:1145-53.

4 Tanamas S, Hanna FS, Cicuttini FM, et al. Does knee malalignment increase the risk of development and progression of knee osteoarthritis? A systematic review. Arthritis Rheum 2009;61:459-67.

5 Duncan R, Peat G, Thomas E, et al. Incidence, progression and sequence of development of radiographic knee osteoarthritis in a symptomatic population. Ann Rheum Dis 2011;70:1944-8.

6 Lankhorst NE, Damen J, Oei EH, et al. Incidence, prevalence, natural course and prognosis of patellofemoral osteoarthritis: the cohort hip and cohort knee study. Osteoarthritis Cartilage 2017;25:647-53.

7 Reeves ND, Bowling FL. Conservative biomechanical strategies for knee osteoarthritis. Nat Rev Rheumatol 2011;7:113-22.

8 Sharma L, Lou C, Cahue S, et al. The mechanism of the effect of obesity in knee osteoarthritis: the mediating role of malalignment. Arthritis Rheum 2000;43:568-75.

9 Rytter S, Jensen LK, Bonde JP, et al. Occupational kneeling and meniscal tears: a magnetic resonance imaging study in floor layers. $J$ Rheumatol 2009;36:1512-9.

10 Jensen LK, Mikkelsen S, Loft IP, et al. Radiographic knee osteoarthritis in floorlayers and carpenters. Scand J Work Environ Health 2000;26:257-62.

11 Block JA, Shakoor N. Lower limb osteoarthritis: biomechanical alterations and implications for therapy. Curr Opin Rheumatol 2010;22:544-50.

12 Englund $\mathrm{M}$. The role of biomechanics in the initiation and progression of oa of the knee. Best Pract Res Clin Rheumatol 2010;24:39-46.

13 Arnold JB, Wong DX, Jones RK, et al. Lateral wedge Insoles for reducing biomechanical risk factors for medial knee osteoarthritis progression: a systematic review and meta-analysis. Arthritis Care Res 2016;68:936-51.

14 Bennell KL, Bowles K-A, Wang Y, et al. Higher dynamic medial knee load predicts greater cartilage loss over 12 months in medial knee osteoarthritis. Ann Rheum Dis 2011;70:1770-4.

15 Brisson NM, Wiebenga EG, Stratford PW, et al. Baseline knee adduction moment interacts with body mass index to predict loss of medial tibial cartilage volume over 2.5 years in knee osteoarthritis. $J$ Orthop Res 2017;35:2476-83.

16 Briem K, Snyder-Mackler L. Proximal gait adaptations in medial knee oa. J Orthop Res 2009;27:78-83.

17 Yang F, Liu W. Knee joint biomechanics of simplified 24 tai chi forms and association with pain in individuals with knee osteoarthritis: a pilot study. Osteoarthr Cartil Open 2021;3. doi:10.1016/j. ocarto.2021.100149. [Epub ahead of print: 0603 2021]

18 Hinman RS, Bowles KA, Bennell KL. Laterally wedged insoles in knee osteoarthritis: do biomechanical effects decline after one month of wear? BMC Musculoskelet Disord 2009;10:146.

19 Divine JG, Hewett TE. Valgus bracing for degenerative knee osteoarthritis: relieving pain, improving gait, and increasing activity. Phys Sportsmed 2005;33:40-6.

20 Lange AK, Vanwanseele B, Fiatarone Singh MA. Strength training for treatment of osteoarthritis of the knee: a systematic review. Arthritis Rheum 2008;59:1488-94.

21 Liu C-ju, Latham NK, Cochrane Bone, Joint and Muscle Trauma Group. Progressive resistance strength training for improving physical function in older adults. Cochrane Database Syst Rev 2009;124:Cd002759.

22 Mikesky AE, Meyer A, Thompson KL. Relationship between quadriceps strength and rate of loading during gait in women. $J$ Orthop Res 2000;18:171-5.

23 Cheung RTH, Ho KKW, Au IPH, et al. Immediate and short-term effects of gait retraining on the knee joint moments and symptoms in patients with early tibiofemoral joint osteoarthritis: a randomized controlled trial. Osteoarthritis Cartilage 2018;26:1479-86.

24 Shull PB, Jirattigalachote W, Hunt MA, et al. Quantified self and human movement: a review on the clinical impact of wearable sensing and feedback for gait analysis and intervention. Gait Posture 2014;40:11-19.

25 Sasaki T, Yasuda K. Clinical evaluation of the treatment of osteoarthritic knees using a newly designed wedged insole. Clin Orthop Relat Res 1987;221:181???187-7.

26 Yasuda K, Sasaki T. The mechanics of treatment of the osteoarthritic knee with a wedged insole. Clin Orthop Relat Res 1987;215:162-72.

27 Hinman RS, Bowles KA, Metcalf BB, et al. Lateral wedge insoles for medial knee osteoarthritis: effects on lower limb frontal plane biomechanics. Clin Biomech 2012;27:27-33.

28 Higgins JPT TJ, Chandler J, Cumpston M. Cochrane Handbook for systematic reviews of interventions version 6.2 (updated February 2021). Cochrane 2021 www.training.cochrane.org/handbook

29 Salanti G, Del Giovane C, Chaimani A, et al. Evaluating the quality of evidence from a network meta-analysis. PLoS One 2014;9:e99682. 
30 Lu G, Ades AE. Combination of direct and indirect evidence in mixed treatment comparisons. Stat Med 2004;23:3105-24.

31 Lu G, Ades AE, Sutton AJ, et al. Meta-Analysis of mixed treatment comparisons at multiple follow-up times. Stat Med 2007:26:3681-99.

32 Smith TC, Spiegelhalter DJ, Thomas A. Bayesian approaches to random-effects meta-analysis: a comparative study. Stat Med 1995:14:2685-99.

33 Caldwell DM, Ades AE, Higgins JPT. Simultaneous comparison of multiple treatments: combining direct and indirect evidence. BMJ 2005;331:897-900.

34 Cooper NJ, Sutton AJ, Morris D, et al. Addressing between-study heterogeneity and inconsistency in mixed treatment comparisons: application to stroke prevention treatments in individuals with nonrheumatic atrial fibrillation. Stat Med 2009;28:1861-81.

35 Dias S, Welton NJ, Caldwell DM, et al. Checking consistency in mixed treatment comparison meta-analysis. Stat Med 2010;29:932-44.

36 Salanti G, Higgins JPT, Ades AE, et al. Evaluation of networks of randomized trials. Stat Methods Med Res 2008;17:279-301.

37 Welton NJ, Caldwell DM, Adamopoulos E, et al. Mixed treatment comparison meta-analysis of complex interventions: psychological interventions in coronary heart disease. Am J Epidemiol 2009;169:1158-65.

38 Turner RM, Jackson D, Wei Y, et al. Predictive distributions for between-study heterogeneity and simple methods for their application in Bayesian meta-analysis. Stat Med 2015;34:984-98

39 Dias S, Welton NJ, Sutton AJ, et al. Evidence synthesis for decision making 4: inconsistency in networks of evidence based on randomized controlled trials. Med Decis Making 2013;33:641-56.

40 Dias S, Welton NJ, Sutton AJ. NICE decision support unit technical support documents. NICE dsu technical support document 2: a generalised linear modelling framework for pairwise and network meta-analysis of randomised controlled trials. London: National Institute for Health and Care Excellence (NICE), 2014.

41 Arazpour M, Bani MA, Maleki M, et al. Comparison of the efficacy of laterally wedged insoles and bespoke unloader knee orthoses in treating medial compartment knee osteoarthritis. Prosthet Orthot Int 2013;37:50-7.

42 Barrios JA, Butler RJ, Crenshaw JR, et al. Mechanical effectiveness of lateral foot wedging in medial knee osteoarthritis after 1 year of wear. J Orthop Res 2013;31:659-64.

43 Bennell KL, Hunt MA, Wrigley TV, et al. Hip strengthening reduces symptoms but not knee load in people with medial knee osteoarthritis and varus malalignment: a randomised controlled trial. Osteoarthritis Cartilage 2010;18:621-8.

44 Bennell KL, Kyriakides M, Metcalf B, et al. Neuromuscular versus quadriceps strengthening exercise in patients with medial knee osteoarthritis and varus malalignment: a randomized controlled trial. Arthritis Rheumatol 2014;66:950-9.

45 Erhart-Hledik JC, Elspas B, Giori NJ, et al. Effect of variable-stiffness walking shoes on knee adduction moment, pain, and function in subjects with medial compartment knee osteoarthritis after 1 year. $J$ Orthop Res 2012;30:514-21.

46 Foroughi N, Smith RM, Lange AK, et al. Lower limb muscle strengthening does not change frontal plane moments in women with knee osteoarthritis: a randomized controlled trial. Clin Biomech 2011;26:167-74.

47 Holsgaard-Larsen A, Clausen B, Søndergaard J, et al. The effect of instruction in analgesic use compared with neuromuscular exercise on knee-joint load in patients with knee osteoarthritis: a randomized, single-blind, controlled trial. Osteoarthritis Cartilage 2017;25:470-80.

48 Hunt MA, Charlton JM, Krowchuk NM, et al. Clinical and biomechanical changes following a 4-month toe-out gait modification program for people with medial knee osteoarthritis: a randomized controlled trial. Osteoarthritis Cartilage 2018;26:903-11.

49 Hunt MA, Pollock CL, Kraus VB, et al. Relationships amongst osteoarthritis biomarkers, dynamic knee joint load, and exercise: results from a randomized controlled pilot study. BMC Musculoskelet Disord 2013;14:115.

50 Jones RK, Nester CJ, Richards JD, et al. A comparison of the biomechanical effects of valgus knee braces and lateral wedged insoles in patients with knee osteoarthritis. Gait Posture 2013;37:368-72.

51 Khosravi M, Arazpour M, Sharafat Vaziri A. An evaluation of the use of a lateral wedged insole and a valgus knee brace in combination in subjects with medial compartment knee osteoarthritis (oa). Assist Technol 2021;33:87-94

52 Lim B-W, Hinman RS, Wrigley TV, et al. Does knee malalignment mediate the effects of quadriceps strengthening on knee adduction moment, pain, and function in medial knee osteoarthritis? A randomized controlled trial. Arthritis Rheum 2008;59:943-51.

53 Hinman RS, Wrigley TV, Metcalf BR, et al. Unloading shoes for selfmanagement of knee osteoarthritis: a randomized trial. Ann Intern Med 2016;165:381-9.

54 Robert-Lachaine X, Dessery Y, Belzile Étienne L, Belzile É L, et al. Three-Month efficacy of three knee braces in the treatment of medial knee osteoarthritis in a randomized crossover trial. J Orthop Res 2020;38:2262-71.

55 Song Q, Shen P, Mao M, et al. Proprioceptive neuromuscular facilitation improves pain and descending mechanics among elderly with knee osteoarthritis. Scand J Med Sci Sports 2020;30:1655-63.

56 Trombini-Souza F, Matias AB, Yokota M, et al. Long-Term use of minimal footwear on pain, self-reported function, analgesic intake, and joint loading in elderly women with knee osteoarthritis: a randomized controlled trial. Clin Biomech 2015;30:1194-201.

57 Wang X, Xie X, Hou M, et al. [Kinetic mechanism of electroacupuncture for stair climbing in knee osteoarthritis patients]. Zhongguo Zhen Jiu 2017;37:1027-34.

58 Cooper NJ, Kendrick D, Achana F, et al. Network meta-analysis to evaluate the effectiveness of interventions to increase the uptake of smoke alarms. Epidemiol Rev 2012;34:32-45.

59 Lambert PC, Sutton AJ, Abrams KR, et al. A comparison of summary patient-level covariates in meta-regression with individual patient data meta-analysis. J Clin Epidemiol 2002;55:86-94.

60 Schmid CH, Stark PC, Berlin JA, et al. Meta-Regression detected associations between heterogeneous treatment effects and study-level, but not patient-level, factors. J Clin Epidemiol 2004;57:683-97.

61 Thompson SG, Higgins JPT. How should meta-regression analyses be undertaken and interpreted? Stat Med 2002;21:1559-73.

62 Bennell KL, Hinman RS. A review of the clinical evidence for exercise in osteoarthritis of the hip and knee. J Sci Med Sport 2011;14:4-9.

63 Fransen M, McConnell S, Harmer AR, et al. Exercise for osteoarthritis of the knee. Cochrane Database Syst Rev 2015;1:Cd004376.

64 Fransen M, McConnell S, Hernandez-Molina G, et al. Exercise for osteoarthritis of the hip. Cochrane Database Syst Rev 2014;21:Cd007912.

$65 \mathrm{Fu} \mathrm{HCH}$, Lie CWH, Ng TP, HC F, TP N, et al. Prospective study on the effects of orthotic treatment for medial knee osteoarthritis in Chinese patients: clinical outcome and gait analysis. Hong Kong Med J 2015;21:98-106.

66 Goh S-L, Persson MSM, Stocks J, et al. Efficacy and potential determinants of exercise therapy in knee and hip osteoarthritis: a systematic review and meta-analysis. Ann Phys Rehabil Med 2019;62:356-65.

67 Maly MR. Abnormal and cumulative loading in knee osteoarthritis. Curr Opin Rheumatol 2008;20:547-52.

68 Erhart-Hledik JC, Mahtani GB, Asay JL, et al. Changes in knee adduction moment wearing a variable-stiffness shoe correlate with changes in pain and mechanically stimulated cartilage oligomeric matrix levels. J Orthop Res 2021;39:619-27.

69 Maleki M, Arazpour M, Joghtaei M, et al. The effect of knee orthoses on gait parameters in medial knee compartment osteoarthritis: a literature review. Prosthet Orthot Int 2016;40:193-201. 